\title{
An Investigation of the Effects of Hippocampal Lesions in Rats on Pre- and Postoperatively Acquired Spatial Memory in a Complex Environment
}

\author{
Gordon Winocur, ${ }^{1,2,3,4 *}$ Morris Moscovitch, ${ }^{1,3,5}$ R. Shayna Rosenbaum, ${ }^{1,6}$ and Melanie Sekeres ${ }^{7}$
}

\begin{abstract}
In previous work, we showed that adult rats that were reared socially for 3 months in a complex (village) environment retained allocentric spatial memory for that environment following hippocampal lesions (Winocur et al., (2005) Nat Neurosci 8:273-275). In the present series of experiments, we showed that 3 months of postoperative rearing did not confer the same benefits (Experiment 1), although hippocampal groups, with or without rearing experience, exhibited spatial learning after extensive training (Experiments 1 and 2). Experiment 3 showed that as little as 2 weeks of preoperative rearing in the village was sufficient to retain acquired spatial memories after hippocampal lesions. Probe testing revealed that, although rats with hippocampal lesions exhibited remarkably good memory for preoperatively learned locations in the village, they were impaired when changes in task demands required flexible use of existing spatial representations. In a direct test of flexibility (Experiment 4), preoperatively reared rats were administered a blockedroutes task in the original learning environment, in which on designated trials, a barrier was placed across one of the direct paths to the goal compartment. On encountering the barrier, control rats consistently selected the next most direct route, whereas rats with hippocampal lesions, despite using spatial strategies, made more errors and took longer to find the goal. The present results confirm that allocentric spatial memories can survive hippocampal damage but they are schematic in nature and less cohesive than those associated with cognitive maps in intact brains. As well, there was evidence that, although different processes are involved in their formation, the schematic memories that were acquired preoperatively and survived hippocampal lesions are essentially the same as those laboriously formed postoperatively after extensive training. $\odot 2010$ Wiley-Liss, Inc.
\end{abstract}

KEY WORDS: hippocampus; consolidation; transformation; complex village; rats

\footnotetext{
${ }^{1}$ Rotman Research Institute, Baycrest Centre, Toronto, Ontario, Canada; ${ }^{2}$ Department of Psychology, Trent University, Peterborough, Ontario, Canada; ${ }^{3}$ Department of Psychology, University of Toronto, Toronto, Ontario, Canada; ${ }^{4}$ Department of Psychiatry, University of Toronto, Toronto, Ontario, Canada; ${ }^{5}$ Department of Psychology, Baycrest Centre, Toronto, Ontario, Canada; ${ }^{6}$ Department of Psychology, York University, Toronto, Ontario, Canada; ${ }^{7}$ Department of Physiology, University of Toronto, Toronto, Ontario, Canada

This study was approved by the Trent University Animal Care Committee and conducted according to the guidelines of the Canadian Council on Animal Care.

Grant sponsor: Natural Sciences and Engineering Research Council of Canada (NSERC); Grant number: 8181-04; Grant sponsor: Canadian Institutes of Health Research Fellowship; Grant number: 181165.

*Correspondence to: Gordon Winocur, Rotman Research Institute, Baycrest Centre, 3560 Bathurst Street, Toronto, ON M6A 2E1, Canada.

Tel: 416-785-2500, Ext. 3592; Fax: 416-785-2474.

E-mail: gwinocur@rotman-baycrest.on.ca

Accepted for publication 11 September 2009

DOI 10.1002/hipo.20721

Published online in Wiley InterScience (www.interscience.wiley.com).
}

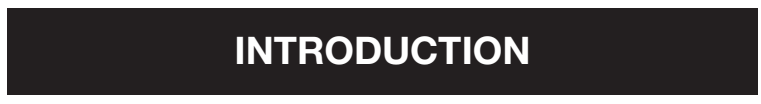

Rats with hippocampal lesions are reliably impaired on conventional tests of spatial learning and memory (O'Keefe and Nadel, 1978; Olton et al., 1979; Morris et al., 1982; Winocur, 1982). However, when allowed to become highly familiar with an environment prior to sustaining hippocampal damage, their spatial memory is remarkably accurate. In a recent study (Winocur et al., 2005), normal adult rats were reared preoperatively in a complex environment (village) with various incentives (e.g., food, water) located in different places. Following surgery, rats with hippocampal lesions remembered previously learned spatial locations based on distal, allocentric spatial relationships as well as control rats, despite being severely impaired in learning new relationships of this kind in the village. These results, which are parallel to similar observations in human amnesics with medial temporal lobe/hippocampal damage (Teng and Squire, 1999; Rosenbaum et al., 2000; Maguire et al., 2006) were taken as evidence that, under certain conditions, the representation of a spatial environment that initially is dependent on the hippocampus can become independent of this structure and represented extra-hippocampally in a form that is capable of supporting accurate navigation (see also Winocur et al., in press).

The purpose of this research was to investigate several questions arising from our previous work (Winocur et al., 2005; Winocur et al., in press). The first relates to whether the savings exhibited by rats with hippocampal lesions were specific to having lived in the village and learned about the environment preoperatively, or simply the result of 3 months experience in the village, regardless of whether it was before the lesion or afterward. If the latter is the case, then rearing rats with hippocampal lesions in the village postsurgery for 3 months would have a similar effect and, in subsequent testing, they should exhibit savings, relative to nonreared, lesioned rats. This and related questions are addressed in Experiments 1 and 2.

A second question relates to the amount of preoperative exposure to the village that is needed for the formation of the extra-hippocampal representation of the spatial environment. In our previous studies (Winocur et al., 2005; Winocur et al., in press), rats 
were reared for 3 months, and we have observed similar savings in rats with hippocampal lesions after 1 month of preoperative exposure (unpublished findings). In Experiment 3 of this research, we ask whether 2 weeks of rearing would be sufficient to yield significant savings.

Finally, although preoperatively reared rats with hippocampal lesions exhibited remarkably good memory for specific locations in the village, in several probe tests they were seen to be less flexible than controls in their use of spatial cues in that environment (Winocur et al., 2005). For example, in one test, the village was rotated $180^{\circ}$ so that the previous relationships between the reward locations and environmental cues were changed. Both control and hippocampal groups eventually learned the new location of the reward, but rats with hippocampal lesions took longer and made many more errors in the process. This is understandable because adapting to this change in orientation requires learning new associations between locations in the village and the extended environment. However, what if it were not necessary to learn new relationships but the task demanded flexible and efficient manipulation of existing representations for successful navigation? In Experiment 4, we asked whether spatial memories that survive hippocampal lesions are as flexible as those of control rats with the same experience. This was accomplished by blocking direct routes to the goal box and assessing the rats' ability to find secondary routes to the reward in what is believed to be a paradigmatic test of hippocampal function (O'Keefe and Nadel, 1978). If preoperatively reared hippocampal rats are less adept in their use of spatial cues, even when they appear to perform normally on some measures, they are likely to be disrupted by this type of manipulation. One of the aims in following this traditional approach to testing spatial memory (Hebb, 1938; Tolman, 1948; O'Keefe and Nadel, 1978) was to gain insight into the nature of the representation that is formed outside the hippocampus and survives damage to the structure.

\section{EXPERIMENT 1}

In our original study (Winocur et al., 2005), rats with hippocampal lesions displayed excellent spatial memory in the village following 3 months of preoperative rearing in that environment. The purpose of Experiment 1 was to determine if 3 months of identical postoperative rearing would confer similar benefits on hippocampally damaged rats. There was evidence in the Winocur et al. (2005) study and others involving humans (e.g., Corkin, 2002) that new spatial learning is possible after hippocampal damage, but it is not clear whether the information acquired and the representations that are formed are similar to those established preoperatively. A second group of rats with hippocampal lesions but with no pre- or postoperative village rearing was also tested for their ability to learn new spatial information, and the performance of the hippocampal groups was compared with that of groups of reared and nonreared control rats.

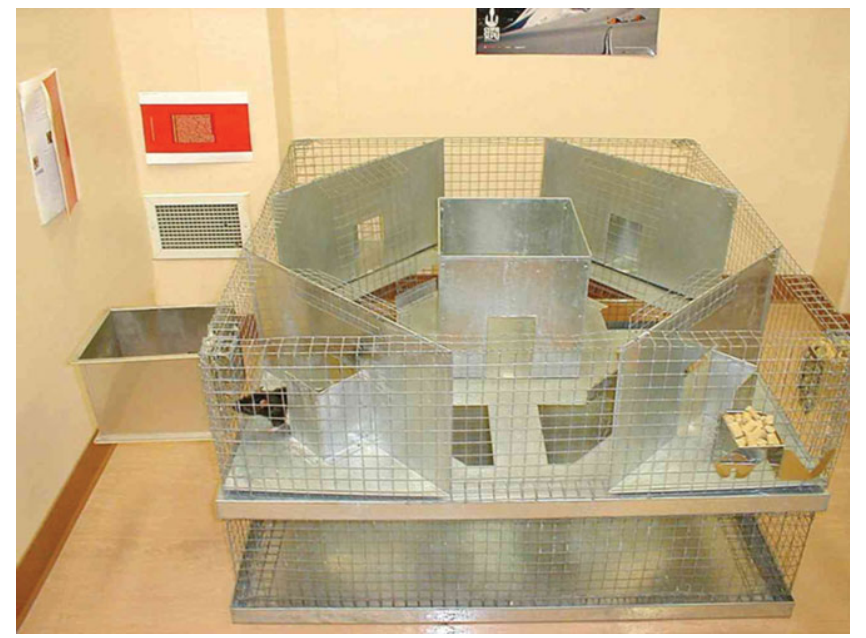

FIGURE 1. The "village" environment. [Color figure can be viewed in the online issue, which is available at www.interscience. wiley.com.]

\section{Materials and Methods}

\section{Subjects}

All experiments in this study were performed on adult male, Long-Evans rats, acquired from Charles River laboratories in St. Constant, Quebec. Food and water were available at all times, except during testing when rats were placed on a $23-\mathrm{h}$ food-deprivation schedule.

All studies were approved by the Trent University Animal Care Committee and the rats were regularly examined by a veterinarian.

Forty rats, $\sim 6$ months old at the beginning of the experiment, successfully completed Experiment 1.

\section{Apparatus}

The village $\left(1.2 \times 1.2 \times 1.2 \mathrm{~m}^{3}\right)$, as shown in Figure 1, was constructed of wire mesh and located in the center of a room with standard laboratory furniture (e.g., desks, book shelves) and pictures on the walls. The room was dimly and uniformly illuminated by overhead lighting. The village contained two levels, with interconnected walkways within and between the levels. Two walkways leading to the lower levels were situated across from the entrance to the reward compartments in the north-east and south-west corners. The walls and ceiling were made of wire mesh, and the walkways of aluminum sheet metal. The upper level, also constructed of sheet metal, consisted of a gathering area in the middle of the upper level with four walls each containing a central opening. This area served as a start box for training and test trials. A compartment, containing food (south-east corner), water (north-west corner), an assortment of toys (north-east corner), or a female rat (south-west corner), was attached to each of four corners on the upper level. 


\section{Village rearing}

Rats in the rearing condition were placed in the village, in groups of seven or eight, and lived there $12 \mathrm{~h} /$ day ( $8 \mathrm{AM}$ to 8 PM) with the room dimly illuminated. During the rearing period, rats were free to explore the entire village, and had free access to the compartments containing the food, water, and toys. The cage containing the female rat was affixed to its compartment and the two were separated by a wire mesh screen. Thus, while the female rat was always present and visible in the south-west compartment, it could not be directly accessed by the other rats. At the end of each daily 12 -h period in the village, the rats were transferred to individual cages in the same room with overhead lighting turned up.

Every 2 or 3 days, with the rats removed from the village, the bedding below the floor was changed and the village was cleaned with a damp cloth, a process that took about $20 \mathrm{~min}$. Between groups, the village was thoroughly washed down.

\section{Memory testing protocol}

Formal memory testing, conducted in dim illumination on groups of seven to eight rats, consisted of five daily trials over 10 days, with an intertrial interval of $\sim 20 \mathrm{~min}$. For each trial, with all the incentives in place, the rat was placed in the start area on the upper level of the village and allowed to find the food compartment. On each trial, the rat was forced to enter the village through a different doorway, thereby ensuring that they followed different routes to the reward compartments. On finding the food compartment, the rat was allowed to eat for 10 s. After each trial, the rat was returned to a holding cage where it awaited the next trial. At the end of each session, rats were returned to their home cages, where they received $20 \mathrm{~g}$ of food.

All intersections of the floors and walkways of the village were used as directional decision points to quantify errors. A rat made an error when it left an intersection in a direction that took it away from the rewarded compartment. The amount of time required to reach the compartment on each trial was also recorded but, as in our previous studies (Winocur et al., 2005; Winocur et al., in press) the latency measures paralleled the error scores. For convenience and ease of comparison with our previous results, with the exception of Experiment 4, we report only the error data in Experiments 1-3. The latency data are available on request. Because of the unique features of the blocked-routes test in Experiment 4, the error and latency scores proved to be informative and so both measures are reported in that experiment.

\section{Surgical and histological procedure}

Surgery was performed on the reared rats within $48 \mathrm{~h}$ of the end of their rearing period. Surgery was performed on the nonreared rats during this time period. For surgery, rats were anesthetized in an induction chamber whose air supply was connected to an isoflurane gas anesthesia machine (Benson Medical Supplies, Markham). The anesthetized rats were positioned in a
Kopf stereotaxic instrument. All lesions were stereotaxically placed with coordinates, based on the Paxinos and Watson (1997) atlas, measured in relation to bregma and the horizontal skull surface. The procedure for making hippocampal lesions was slightly modified from the technique developed by Jarrard and Meldrum (1993). Using a small dental burr, eight holes were drilled through the skull directly above the hippocampus in each hemisphere. For hippocampal groups, hippocampal damage was produced by 10 intracranial microinjections of a solution containing the cellular neurotoxin, NMDA $(5 \mathrm{mg} / \mu \mathrm{L}$ phosphate buffer per site) into each hemisphere. The coordinates were: anterior/posterior: $-3.1,3.1,4.1,4.1,5,5,5,5.8$, $5.8,5.8 \mathrm{~mm}$; lateral: $\pm 1,2.2,2.2,3.5,3,5.2,5.2,4.4,5.1$, $5.1 \mathrm{~mm}$; ventral: $-3.6,3.6,4.4,4.4,4.1,5,7.3,4.4,6.2,7.5$ $\mathrm{mm}$. The solution was infused at a rate of $0.4 \mu \mathrm{L} / \mathrm{min}$ through 30 -gauge stainless steel needles for $38 \mathrm{~s}$, using a $10-\mu \mathrm{L}$ syringe attached to a motorized infusion pump. The last two ventral hippocampal sites were injected for 2 min each. The needles were removed $2 \mathrm{~min}$ after each injection. Control groups were similarly anesthetized, and incisions and burr holes were identical to the lesioned animals with the exception that there was no penetration of brain tissue. All animals were given injections of diazepam $(10 \mathrm{mg} / \mathrm{kg}$ i.p.) during surgical recovery.

On completion of behavioral testing, rats with hippocampal lesions were deeply anesthetized with sodium pentobarbital and perfused with $0.9 \%$ saline followed by $10 \%$ formalin. The fixed brains were removed from the skull and stored in $10 \%$ formalin. Brains were transferred to a $30 \%$ buffered sucrose solution $48 \mathrm{~h}$ prior to sectioning. The brains were then frozen and sliced at $40 \mu \mathrm{m}$. Every fifth section was mounted on gelled glass slides and stained with formal-thionin.

\section{Procedure}

There were four groups of rats in this experiment: hippocampal + rearing (HPC-R [Post], $n=8)$; HPC + nonrearing (HPC-NR, $n=17$ ); operated control $+\mathrm{R}$ (OC-R [Post], $n=$ 6). A group of nonreared, operated controls (OC-NR, $n=9)$ was tested as part of our previous study (Winocur et al., 2005) and, for comparison purposes, their data are included here. It is important to emphasize that these rats were tested only a few months before this study began and that, in every respect, they were treated in exactly the same way as the other rats (see Fig. 2 for design and timelines).

Hippocampal or control surgery was performed according to the procedures described earlier. After a 7-day recovery period in individual cages, rats were placed in the appropriate environment which, for rats in the reared groups, was the village. For 3 months, rats in the rearing condition were treated as described earlier. Rats in the nonreared groups were housed individually in their home cages in another room that was similarly illuminated. These rats were handled for a few minutes every morning.

After 3 months, the village-reared rats were transferred permanently to the individual cages and, along with the nonreared rats in their individual cages, were placed on a food restricted 
Experiment 1

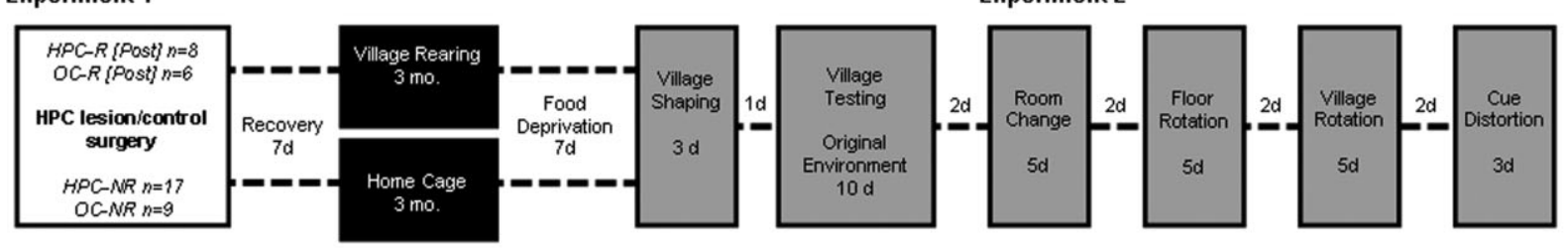

Experiment 3
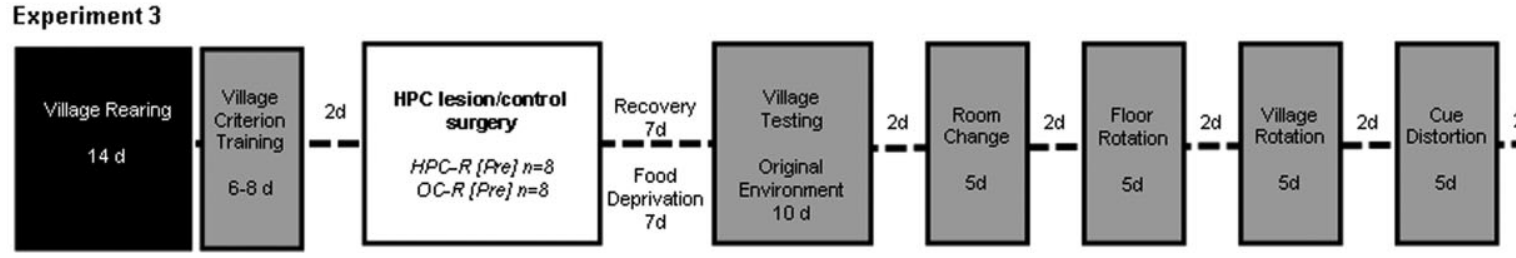

Experiment 4

FIGURE 2. Graphic showing the design and timelines of Experiments 1-4. Note that the OC-NR group in Experiment 1 was tested a few months before the other groups in this experiment and were not administered the probe tests of Experiment 2.
In the latter experiment, the comparisons of principal interest are between the HPC-R [Post] and the OC-R groups, and between the HPC-R [Post] and HPC-R groups. schedule in which they were given $20 \mathrm{~g}$ of standard laboratory chow per day for 7 days.

On the eighth postoperative day, preliminary shaping began in which pairs of rats were placed in the village and allowed to explore freely for $1 \mathrm{~h}$. Rewards were located in the compartments as described earlier. One such session was provided each day for 3 consecutive days and rats were fed at the end of each session. Beginning on the fourth day, rats received 10 days of formal testing in which their ability to find the food compartment was assessed. Testing procedures were as described earlier in the "Memory testing protocol" section.

\section{Data analysis}

Error scores were calculated for each rat by dividing the total number of errors each day by five-the number of daily test trials. Largely because of the excellent performance of some groups after 1 or 2 days of testing in all tests of the various experiments, error scores varied considerably across groups and days. To stabilize the variance and minimize heterogeneity of variance, we used the natural log transform of the error count, incremented by 0.1 to avoid undefined $\log$ of zero. Thus transformed, the outcome measure no longer suffered from constricted variance near the floor of the outcome space. Analyses of variance (ANOVA), with lesion and rearing as betweengroup variables and day as a within-group variable, were performed on the transformed error scores.

\section{Results and Comment}

As can be seen in Figure 3, there was a strong main effect of lesion as rats with hippocampal lesions generally made many more errors than the controls over the 10 -day test period, $F_{1,35}$ $=78.10, P<0.0001$. There were also significant main effects of rearing, $F_{1,35}=12.83, P<0.001$, and day, $F_{9,115}=27.20$, $P<0.0001$. Further analyses revealed a significant effect of rearing in the OC groups, $F_{1,13}=11.36, P<0.005$, indicat- ing that the OC-R [Post] rats benefited from their experience in the village and performed better than their nonreared counterparts. However, although the HPC-R [Post] group made numerically fewer errors than the HPC-NR group on days $1-3$ of testing, neither the rearing $\mathrm{x}$ day interaction, $F<1$, nor the main effect of rearing, $F_{1,22}=2.17, P>0.15$, was statistically significant in rats with hippocampal lesions.

ANOVA also showed the OC groups improved significantly over the 10 days of testing, $F_{9,117}=15.34, P<0.0001$. As

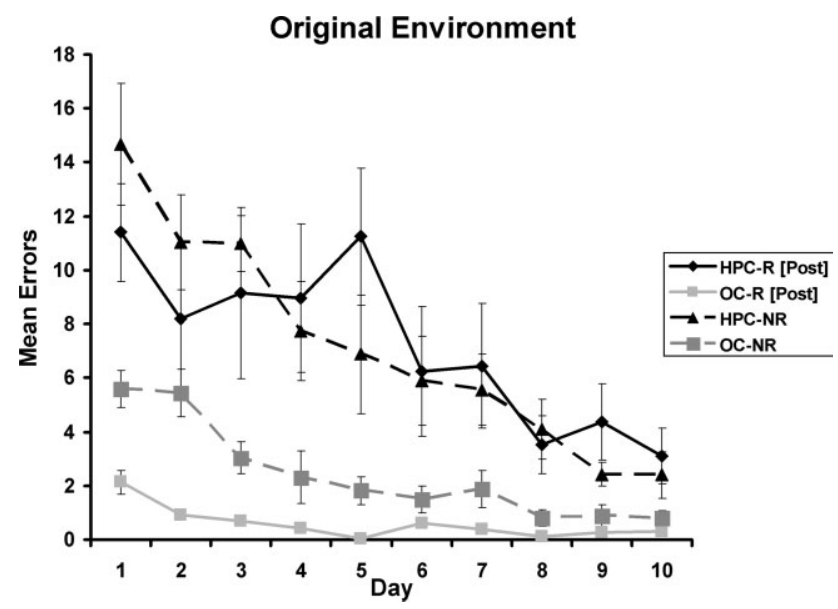

FIGURE 3. Mean number of errors made in finding the reward location in the village in Experiment 1, by hippocampal and control groups that received 3 months of postoperative rearing in the village (HPC-R [Post] and OC-R [Post] groups) or no-rearing (HPC-NR and OC-NR groups). The OC-NR group was tested as part of our original study (Winocur et al., 2005) under conditions identical to those followed in this study. The results show the predicted benefits of rearing in control rats. The hippocampal groups were severely impaired in locating the reward, with no difference between reared and nonreared groups. Error bars refer to standard error of mean (SEM). 
well, even though the HPC-R [Post] and HPC-NR groups performed worse than the $\mathrm{OC}$ groups and did not differ from each other, both HPC groups exhibited significant learning over the same period, $F_{9,198}=11.77, P<0.0001$.

The primary purpose of Experiment 1 was to determine if 3 months of rearing in the village following hippocampal lesions was as effective as 3 months of preoperative rearing in producing a schematic map of the environment in extra-hippocampus structures. The results clearly showed that was not the casewhen tested following the rearing experience the HPC-R [Post] group performed much worse than the preoperatively reared HPC group in the Winocur et al., (2005) study. In that study, the preoperatively reared HPC group performed as well as similarly reared controls. In this study not only did the HPC-R [Post] group perform worse than reared controls, they were no better than the HPC-NR group that had received no prior rearing in the village. Although the HPC groups in this study were severely impaired in finding the food compartment during testing, both lesioned groups showed substantial learning over the 10-day test period. In the next experiment, we sought to understand the nature of the spatial representation and strategies that were acquired by the lesioned rats.

\section{EXPERIMENT 2}

In previous work (Winocur et al., 2005), a series of probe tests showed that both preoperatively reared hippocampallesioned rats, and operated controls, used spatial, rather than nonspatial landmarks or sensory cues, to navigate the village. In Experiment 2, we administered the critical probe tests to the HPC-R [Post] group, a subset of the HPC-NR group, and the OC-R [Post] group of Experiment 1. The purpose was to determine if rats with hippocampal lesions, that experienced the village for the first time postoperatively, also formed spatial representations comparable with those formed by rats reared preoperatively, but used them less efficiently.

In the first test (room-change), the village was moved to a different room and the animals' ability to find the food was tested. If, during original learning, rats had used local or sensory cues in the village, there should be little effect of moving the village to another room. However, if rats used allocentric spatial cues to establish their representation of the original village environment, some disruption would be expected when tested in a different room. For the second test (floor-rotation), the village was returned to the original room but the floor was rotated by $180^{\circ}$, while holding constant the location of the reward compartments. In this test, major disruption in finding the food compartment would be expected if rats had used local or sensory cues during original learning, since only the relationship of these cues to the food compartment had changed. For the third test (village-rotation), the entire village was rotated $180^{\circ}$, relative to the distal cues in the original environment. If rats had used local or sensory cues during original learning, there should be no effect of this manipulation since the relationships between these cues to the food compartment remained constant. However, if rats had used allocentric spatial cues during original learning, they should exhibit considerable negative transfer in this test, since successful performance would depend on learning new spatial relationships. Finally, the rats were tested in the same room but with only a few of the distal cues, in their original location (cue-distortion). Our previous work showed that preoperatively reared rats with hippocampal lesions used the few familiar spatial cues that were still available and navigated the village as efficiently as similarly reared controls (Winocur et al., 2005). The question here was how the HPC-R [Post] and HPC-NR groups of Experiment 1 would perform under these conditions.

\section{Materials and Methods}

\section{Subjects}

The HPC-R [Post] $(n=8)$, HPC-NR $(n=9)$, and OC-R [Post] $(n=6)$ groups in this study were comprised of rats that had participated in Experiment 1. Probe test data were not available for the OC-NR group of Experiment 1. Since the principal comparisons for Experiment 2 are between the HPC-R [Post] and the OC-R groups, and between the HPC-R [Post] and HPC-R groups, these data were not considered essential.

The rats were housed in individual cages in a room that was controlled by a $12 \mathrm{~h}$ light:dark cycle in which the room was dimly illuminated between 8:00 AM and 8:00 PM, and fully illuminated between 8:00 PM and 8:00 AM. Water was always available but, throughout the experiment, rats were maintained on a food-restricted schedule in which they were fed $20 \mathrm{~g}$ of standard rat chow each day following testing. All testing was conducted during the dimly illuminated phase of the cycle.

\section{General procedure}

The rats were maintained on a food-restricted schedule and probe testing for Experiment 2 began a few days after the completion of Experiment 1. Testing procedures were identical to those followed in previous studies (Winocur et al., 2005; Winocur et al., in press), and in Experiment 1. Two days separated each testing condition. For all tests, rats received five trials/day for five consecutive days, except in the cue-distortion condition where, because of a scheduling problem, the rats received 3 days of testing (see Fig. 2 for a summary of sequence and timeline of tests.).

\section{Room-change}

A few days after completion of Experiment 1, the village was relocated to a different room. The new room had an entirely different array of cues but the reward compartments were located in the same relationship to the village and to each other (e.g., the food compartment was in the south-east corner of the village and the water compartment in the north-west corner).

\section{Floor-rotation}

For the floor-rotation condition, the village was returned to the same place in the original room. The inner floors and walk- 
A

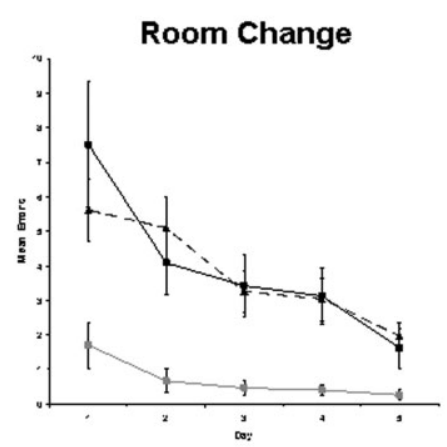

C

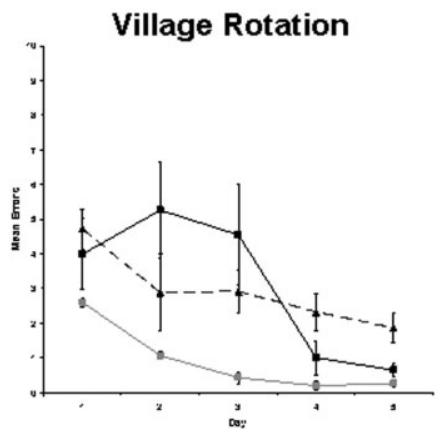

FIGURE 4. Mean numbers of errors made by HPC-R [Post], OC-R [Post], and HPC-NR groups of Experiment 1 in the probe tests of Experiment 2. All groups exhibited an initial increase in errors in the room-change and village-rotation tests, where the goal box was aligned with different sets of spatial cues, but the

ways within the village were rotated $180^{\circ}$ but the reward compartments remained in their original locations.

\section{Village-rotation}

Following the floor-rotation testing, the floors and walkways were returned to their original positions. For the village-rotation test, the entire village was rotated $180^{\circ}$. In this configuration, all the reward compartments were in a different location relative to distal cues in the room. Thus, for example, the food compartment, which had always been in the south-east corner was now located in the north-west corner, and the water compartment, which had been in the north-west corner was now in the south-east corner, and so on.

\section{Cue-distortion}

For the cue-distortion condition, the original environment test room was reconfigured. Some equipment and a desk that were part of the original environment were removed from the test room. In addition, several small pieces of furniture (e.g., chair, table) were relocated in the room. Other pieces (e.g., stool) and wall fixtures (e.g., light switch) remained in their original places. New furniture (e.g., bookcase) was brought in and replaced previous objects or occupied new places. There was a general reorganization of the wall posters, with some
B
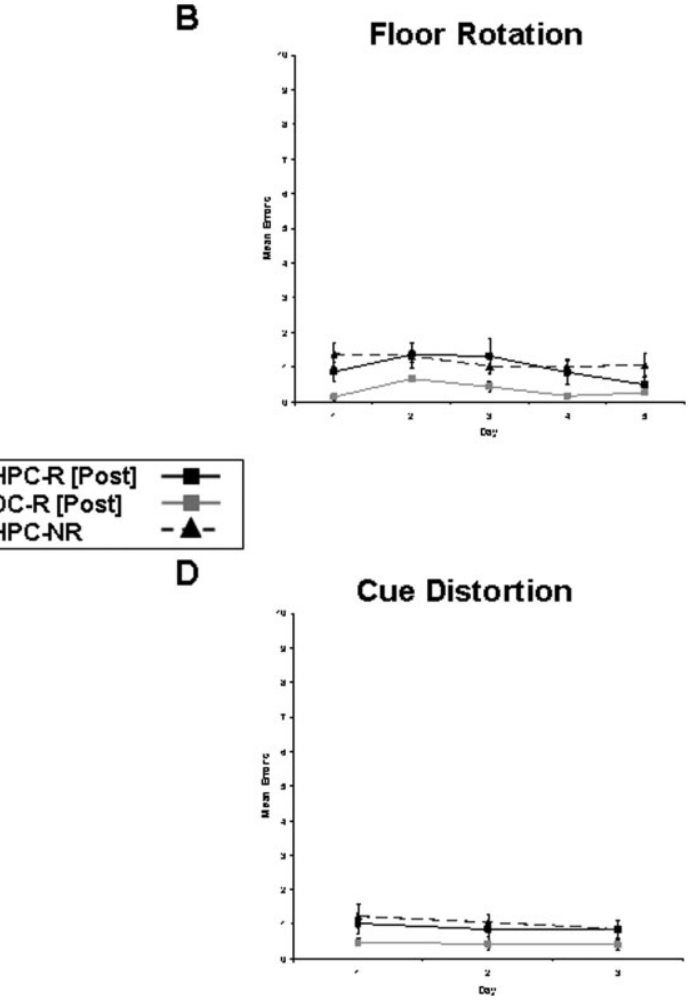

effect was greater in the HPC groups. HPC and OC groups performed well and with virtually no difference in the floor-rotation and cue-distortion tests, where spatial relationships were the same as in original learning. Error bars refer to SEM.

retained in their original places, others relocated or removed, and a few new pictures added. The village remained in its original location and orientation.

\section{Results}

The results for this experiment are graphically presented in Figure 4.

\section{Room-change}

Figure $4 \mathrm{~A}$ shows that all three groups made more errors on day 1 than they did at the completion of testing in the original environment of Experiment 1 (see Fig. 3), and that the effect was especially marked in the HPC groups. Rats in the OC-R [Post] group recovered quickly and by day 2 were making an average of less than one error over the five trials of daily testing. By comparison, the HPC groups consistently performed worse than the OC-R [Post] group, $F_{2,19}=17.90, P<$ 0.0001 , and did not reestablish their optimal performance levels until day 5 . This group effect was due to significant differences between the OC-R [Post] and HPC-R [Post] groups, $F_{1,13}=19.01, P<0.001$, and between the OC-R [Post] and HPC-NR, $F_{1,11}=76.08, P<0.0001$, groups. The reared and nonreared HPC groups did not differ from each other on this test, $F<1$. 


\section{Floor-rotation}

Figure $4 \mathrm{~B}$ shows that both HPC groups transferred well to the floor-rotation test, making an average of about one error/ day, even on the first day of testing. Nevertheless, overall, the difference in error scores between the HPC and OC-R [Post] groups proved to be statistically significant, $F_{2,19}=3.93, P<$ 0.05 .

\section{Village-rotation}

Figure $4 \mathrm{C}$ shows that, in the village-rotation condition, both HPC groups performed worse than the OC-R [Post] group, particularly over days $1-3$. ANOVA applied to these data revealed a significant group $\mathrm{x}$ day interaction, $F_{4,44}=4.83$, $<0.003$. Further analyses indicated that the OC-R [Post] group differed significantly from both the HPC-R [Post], $F_{1,13}=$ 14.41, $P<0.002$, and HPC-NR groups, $F_{1,11}=48.00, P<$ 0.0001 , groups. The HPC groups did not differ from each other on this test, $F_{1,14}=1.49, P>0.20$.

\section{Cue-distortion}

Figure 4D shows that all groups performed well in the cuedistortion test. ANOVA applied to these data yielded no significant effect of group, $F_{2,19}=1.82, P>0.15$.

\section{Discussion of Results of Experiments 1 and 2}

The results of Experiments 1 and 2 clearly established that, in terms of spatial learning and memory, the effects of rearing rats in the village for 3 months are quite different, depending on whether the rats are reared before or after hippocampal lesions. When preoperatively reared, rats with hippocampal lesions exhibited excellent spatial memory for a previously learned spatial location, and when measured by the number of errors made, performed as well as similarly reared controls (Winocur et al., 2005). In contrast, in Experiment 1 of this study, postoperatively reared hippocampal rats failed to show significant savings and performed as poorly as rats with hippocampal lesions that had no previous experience with the village.

Despite their poor performance, both HPC groups in Experiment 1 showed significant learning over the 10-day test period. Since the HPC groups did not differ from each other on this test, the improvement exhibited by the HPC-R [Post] group cannot be attributed to savings resulting from its rearing experience. Rather, this appears to be new goal-directed, spatial learning of which both HPC groups were equally capable.

Taken together, the results indicate that rearing rats with hippocampal lesions in a complex environment even for an extended period is not sufficient to support the formation of a spatial representation of that environment. To achieve this, specialized training is required. By comparison, exposure to the environment for an extended period is sufficient for normal rats to form a detailed representation that can survive hippocampal damage (Winocur et al., 2005; Winocur et al., in press). Experiment 2 assessed the nature of the representation that was formed postoperatively in extra-hippocampal structures to determine whether they resembled the preoperatively formed representations that survived hippocampal damage.

Probe testing in Experiment 2 indicated that both HPC groups used allocentric spatial cues in learning the location of food during testing in the original environment (Experiment 1). Their equally poor performance in the room-change and village-rotation tests and, by comparison, their excellent performance in the floor-rotation and the cue-distortion tests, parallel that of the preoperatively reared HPC and control rats in the Winocur et al. (2005) study and the OC-R group in Experiment 1 of this study. It may be noted that, in the floorrotation test, the HPC groups performed slightly worse than the OC-R group. The difference, although small, was statistically significant. In this test and in the cue-distortion test, both HPC groups performed much better than in the room-change and the village-rotation tests, where they had to learn new spatial relationships to find the reward location. Our view is that the group difference in the floor-rotation test, while statistically significant, is not meaningful, and probably represents a nonspecific effect of the hippocampal lesion.

This general pattern indicates that performance was disrupted only when the relationship between allocentric spatial cues and the reward location was changed and that rats with hippocampal lesions used an allocentric spatial strategy to navigate the village, regardless of their rearing experience. Because performance in the cue-distortion condition was virtually unaffected by a reorganization of environmental cues, it appears that a minimal number of cues that retain the original configuration, perhaps only the room's geometry, is sufficient to support navigation.

While the similar pattern of performance among all groups suggests that rats with hippocampal lesions used allocentric spatial representations to find the reward compartment, there were indications in Experiment 2 that the spatial strategies, or representations, were different. For example, in the room-change and village-rotation probe tests, after using a particular configuration of allocentric cues to learn the location of the food, rats had to deal subsequently with a different configuration to find the food. Control rats adapted quickly to the change in both tests and, by day 2 or 3 , were performing at optimal levels. Rats with hippocampal lesions had much more difficulty and did not re-establish optimal performance levels until day 5 . Winocur et al. (2005) observed the same effect and suggested that the spatial memory of the preoperatively reared hippocampal rats was supported by a schematic cognitive map that represented only coarse features of the environment. Such coarse features make it difficult to remap new precise relationships between specific locations in the village and the broader environment. This schematic representation resides in extra-hippocampal, neocortical structures and, while it is less efficient than a cognitive map that depends on the hippocampus, it is capable of supporting spatial navigation but to a more limited degree. The results of Experiments 1 and 2 suggest that rats with hippocampal lesions are also capable of spatial learning and that such learning may be based on the formation of similar, sche- 
matic representations in extra-hippocampal structures. Some implications of this interpretation are examined in Experiments 3 and 4, and discussed further in the "General Discussion" section.

\section{EXPERIMENT 3}

The purpose of Experiment 3 was to determine if 2 weeks of continuous rearing in the village, preoperatively, is sufficient to yield the same level of performance previously seen in rats with hippocampal lesions that, prior to surgery, had spent 12 $\mathrm{h} /$ day in the village for 3 months (Winocur et al., 2005) or 1 month (unpublished observations). Following the standard test of spatial memory in the original environment, rats were administered the probe tests of Experiment 2 to assess the strategies employed in navigating the village.

\section{Materials and Methods}

Sixteen adult male, Long-Evans rats participated in this experiment. All rats were subjected to 2 weeks of continuous rearing in the village in groups of five or six rats. Except for the continuous rearing and the duration of the rearing period, rearing conditions were identical to those of Experiment 1 and our previous studies (Winocur et al., 2005; Winocur et al., in press). Every other day, the rats were removed, the bedding changed, and the village wiped down with a wet cloth.

After 2 weeks in the village, following our standard procedure, rats received 10 training trials/day to establish their ability to find the food compartment to a criterion of $80 \%$ errorless trials over 2 days. Within $48 \mathrm{~h}$ of the completion of training, rats were randomly assigned to HPC-R [Pre] $(n=8)$ and OC-R [Pre] $(n=8)$ groups, and surgery was performed as reported previously. One week after surgery, rats were placed on foodrestriction for 7 days in preparation for memory testing. Postoperative testing procedures were identical to those reported in Experiments 1 and 2. Spatial memory was initially tested in the original environment, followed by probe tests in the following order: Room-change, floor-rotation, village-rotation, and cuedistortion. One rat in the HPC-R [Pre] group sustained an injury to its foot after the floor-rotation test and could not be tested again. Histology performed on that rat's brain indicated a typical lesion and so the collected data are reported (see Fig. 2 for a summary of sequence and timeline of tests.).

\section{Results and Comment}

\section{Original-environment}

Preoperatively, rats required 6-8 days to reach criterion and there was no difference between the rats assigned to the two groups. The postoperative error scores of the OC-R [Pre] and HPC-R [Pre] groups over 10 days of testing in the original environment are presented in Figure 5A. ANOVA applied to these data indicated that neither the group x day interaction, $F_{9,26}<$
1 , nor the main effect of group, $F_{1,14}=2.37, P>0.10$, was statistically significant. Moreover, their performance was very similar to that of hippocampal and control groups tested in the same way after 3 months of rearing (Winocur et al., 2005) and much better than OC and HPC groups that received no rearing (Winocur et al., 2005; Experiment 1 of this study). The results indicate that 2 weeks of continuous preoperative rearing in the village led to substantial and comparable savings in rats that subsequently received hippocampal lesions or control surgery.

\section{Room-change}

Figure $5 \mathrm{~B}$ shows that both groups made more errors on day 1 than they did at the completion of testing in the original environment (Fig. 5A), and that the effect was especially marked in the HPC groups. Both groups improved over the 5 days of testing, $F_{4,56}=16.19, P<0.0001$, but the HPC-R [Pre] group consistently performed worse than the OC-R [Pre] group, $F_{1,14}=19.81, P<0.001$. The group $\mathrm{x}$ day interaction was not statistically significant, $F_{4,56}=1.49, P>0.20$.

\section{Floor-rotation}

Figure $5 \mathrm{C}$ shows that both groups transferred well to the floor-rotation test, but the HPC-R [Pre] group consistently made slightly more errors than the OC-R [Pre] group, $F_{1,14}=$ 7.96, $P<0.02$.

\section{Village-rotation}

Figure 5D shows that both groups initially made more errors in the village-rotation condition than they did at the end of testing in the original-environment condition but that the OC$\mathrm{R}$ [Pre] group eventually recovered to its optimal level of performance. The HPC-R [Pre] made many more errors and showed little improvement over 5 days of testing. ANOVA indicated a highly significant group difference, $F_{1,14}=18.48$, $P<0.001$.

\section{Cue-distortion}

Figure 5E shows that both groups performed well in the cue-distortion test. ANOVA applied to these data yielded no significant effect of group, $F_{1,13}<1$.

The results of Experiment 3 confirm that familiarity with a complex environment can produce spatial memories that survive bilateral hippocampal damage (Winocur et al., 2005), and that as little as 2 weeks experience is sufficient for this effect to occur. It is also noteworthy that the HPC-R [Pre] group in this experiment performed as well as rats with hippocampal lesions that received 3 months of preoperative rearing in the village (Winocur et al., 2005). At the same time, despite the excellent performance of the HPC-R [Pre] group in the original-environment test, group differences in the room-change and villagerotation tests provide further evidence that the hippocampal rats' spatial memories were relatively inflexible and that their ability to use highly familiar spatial cues for new learning was impaired. In this regard as well, the 2-week group resembled 

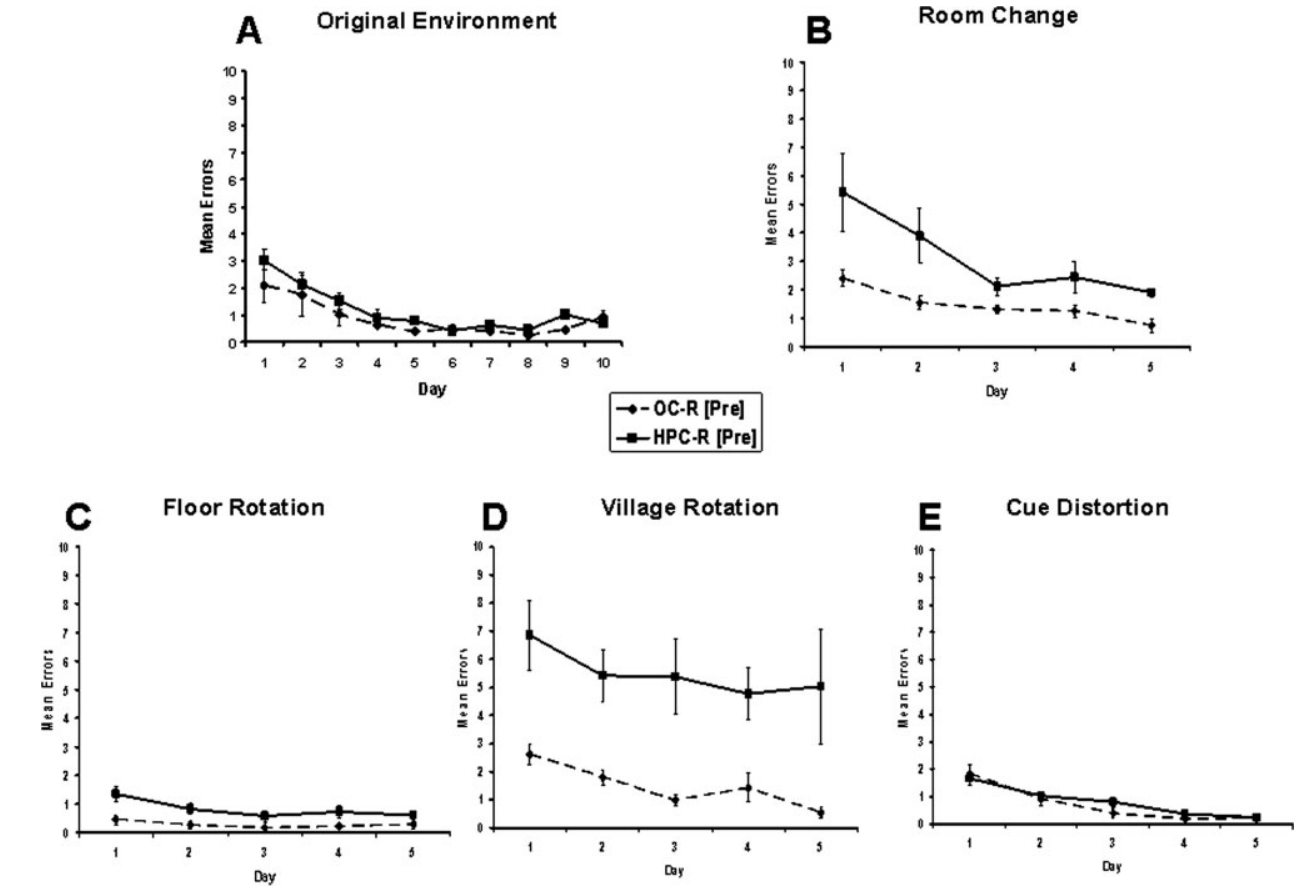

FIGURE 5. Mean number of errors to find the reward location in the village in Experiment 3, by hippocampal and control groups that received 2 weeks of preoperative rearing in the village (HPC$R$ [Pre] and OC-R [Pre] groups), when tested in the original envi-

ronment $(\mathrm{A})$, and in the various probe tests $(\mathrm{B}-\mathrm{D})$. The results are virtually identical to those reported previously for rats with hippocampal lesions receiving 3 months of preoperative rearing in the village (Winocur et al., 2005). Error bars refer to SEM.

rats with hippocampal lesions that had been reared preoperatively for 3 months.

\section{EXPERIMENT 4}

The aims of Experiment 4 were: (1) to conduct a direct test of the flexibility of preoperatively formed spatial memories in the HPC-R [Pre] and the OC-R [Pre] groups of Experiment 3, and (2) to obtain insight into the nature of the memory representation that survived hippocampal damage and guided spatial navigation in the HPC-R [Pre] group. In this experiment, rats were tested in the original village environment under two conditions. On half of the daily trials, they were required to find the food compartment following standard testing procedures, while on the other half of the trials there was a barrier blocking one of the most preferred and direct routes.

We chose the blocked-routes task because traditionally it has been considered a test of the animal's use of a spatial cognitive map in navigating a complex environment (Hebb, 1938; Tolman, 1948). The rationale is that such a map enables animals to navigate efficiently in the environment and provides the flexibility needed to adjust to changes, because the representation is cohesive (well-integrated) and embodies the relations that all elements of the map bear to each other. The prediction, based on Tolman's work, was that on encountering a barrier that blocked the most direct route to its goal, OC-R [Pre] rats would find the next, shortest route. Would the same be true of the HPC-R [Pre] group whose postoperative navigation in the original village was excellent and indistinguishable from that of the OC-R group? We speculated that, once the hippocampus was removed, navigation would be based on a schematic, coarse representation of the environment mediated by extra-hippocampal structures. One possibility is that such a representation retains sufficient information to allow for flexible adjustments to changes in the original environment, as long as the relationships among crucial elements in the environment are fixed. On this view, the HPC-R [Pre] group would perform well in the blocked-routes task. An alternative outcome, suggested by findings in the room-change and village-rotation probe tests, is based on the idea that the representation of the environment in the brains of rats with hippocampal lesions consists of collections of poorly integrated elements that preclude flexible remapping when changes to the environment are introduced. On this view, it is predicted that, on encountering an obstacle en-route to the goal, rats in the HPC-R [Pre] group would have difficulty re-orienting to new spatial reference points even when external relationships in the environment remain unchanged. The result would be that the HPC-R [Pre] group would be worse than controls at finding secondary routes to preoperatively learned locations.

\section{Materials and Methods}

The rats that completed Experiment 3 also participated in Experiment 4, with the exception of one rat in the HPC-R [Pre] group that died just before the beginning of Experiment 4. 


\section{Village: Barrier Study}
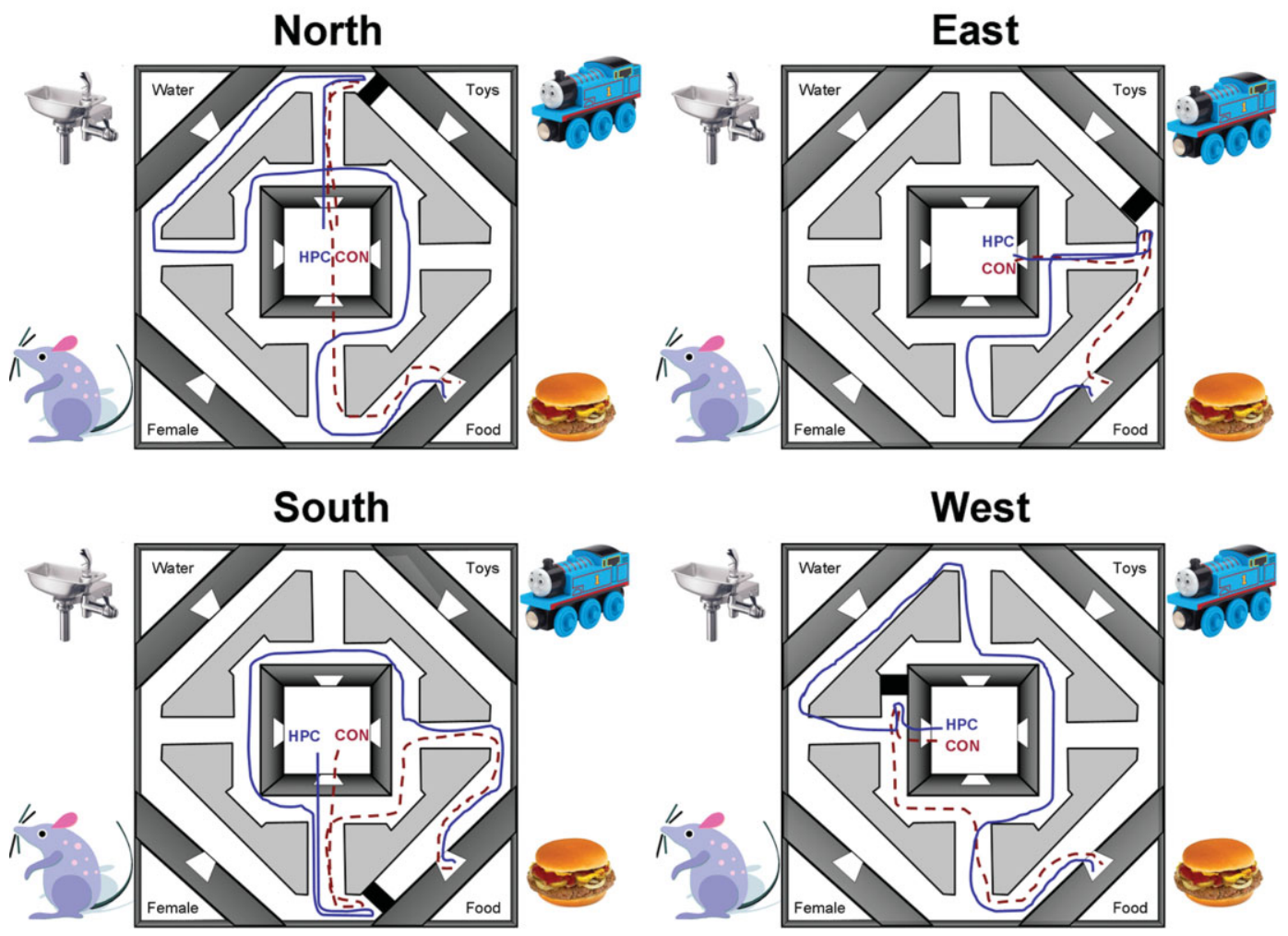

FIGURE 6. Diagrammatic aerial views of the village indicating representative locations of the barriers (black bar) for each compass point, along with typical re-routing paths to the food compartment taken by the HPC-R [Pre] (solid lines) and OC-R [Pre] (broken lines) groups of Experiment 3, after encountering the barrier in

Rats remained on food restriction following the completion of Experiment 3 and 2 days later Experiment 4 began. Testing consisted of 8 trials/day-without a barrier (no-barrier trials) on the four odd-numbered trials and with a barrier (barrier trials) on the four even-numbered trials. Testing procedures for the barrier and no-barrier trials were identical to those described previously, with records kept on each trial of the rat's path, latency to reach the food compartment, and the number of errors made in the process. On every day, each of the four compass-point exit doors ( $\mathrm{N}, \mathrm{E}, \mathrm{S}, \mathrm{W})$ was used once as the starting point for each of the four barrier and four no-barrier trials.

For each barrier trial, the barrier, which consisted of a transparent Plexiglas panel $(15 \mathrm{~cm}$ high), blocked the path along one of the preferred routes between the north, east, south, or west door in the start area and the food compartment. There were two barrier locations for each compass point-one just past the perimeter turn and before the food compartment, and the other just past the first turn from the door. These locations were selected because they are direct and frequently chosen paths by reared HPC and control groups when they are postoperatively tested in the same environment. Figure 6 indicates representative locations of the barriers for each compass point,

Experiment 4. Both groups were efficient at finding a secondary path and appeared to be guided by a spatial strategy, although control rats were more likely to find the next most direct path to the goal. Error bars refer to SEM. [Color figure can be viewed in the online issue, which is available at www.interscience.wiley.com.]

along with typical re-routing paths taken by rats in the HPC (solid lines) and control groups (broken lines) after encountering a barrier. The barriers were positioned according to a schedule that ensured that (1) a barrier was located at each compass point once per test session, (2) a barrier was placed at each location four times over the 8 days of testing, and (3) the order of compass-point locations for the even-numbered barrier trials changed each day (as it did for the no-barrier trials).

\section{Results and Comment}

Neither the compass point nor the location of the barrier at each compass point affected performance of the HPC and control groups on the barrier trials. Accordingly, for purposes of analysis and to simplify presentation, the data were collapsed across the compass points and combined into single data sets for trials in which a barrier was present or absent.

Figure 7 presents the groups' performance on the barrier (Fig. 7A) and no-barrier (Fig. 7B) trials in terms of mean number of errors and mean latency per trial, across the 8 days of testing. The performance of the OC-R [Pre] group on the barrier and no-barrier trials was virtually the same as when they were tested in the original-environment test where the relation- 


\section{Barrier}

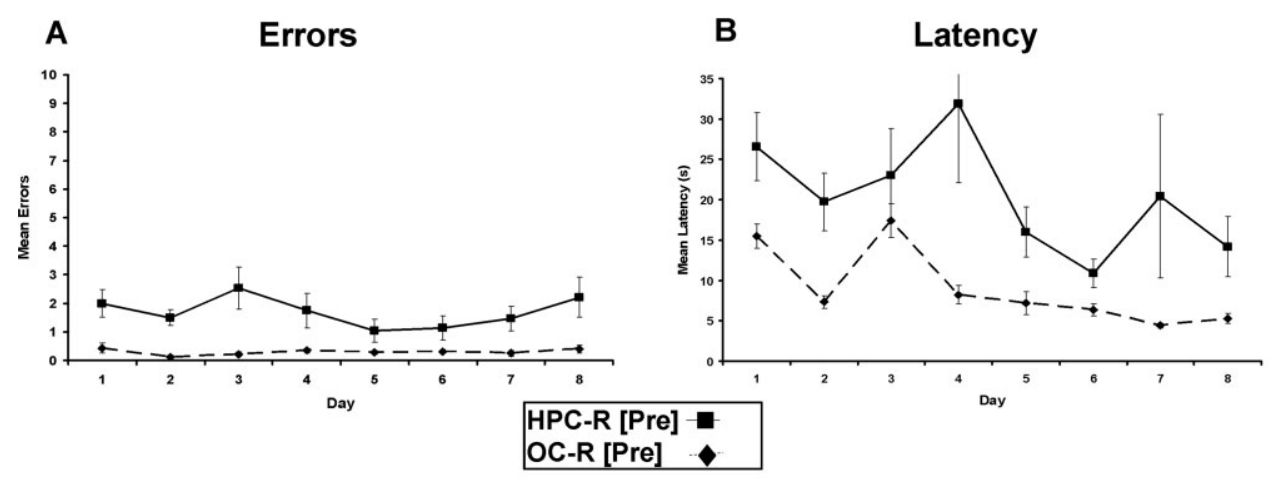

No Barrier
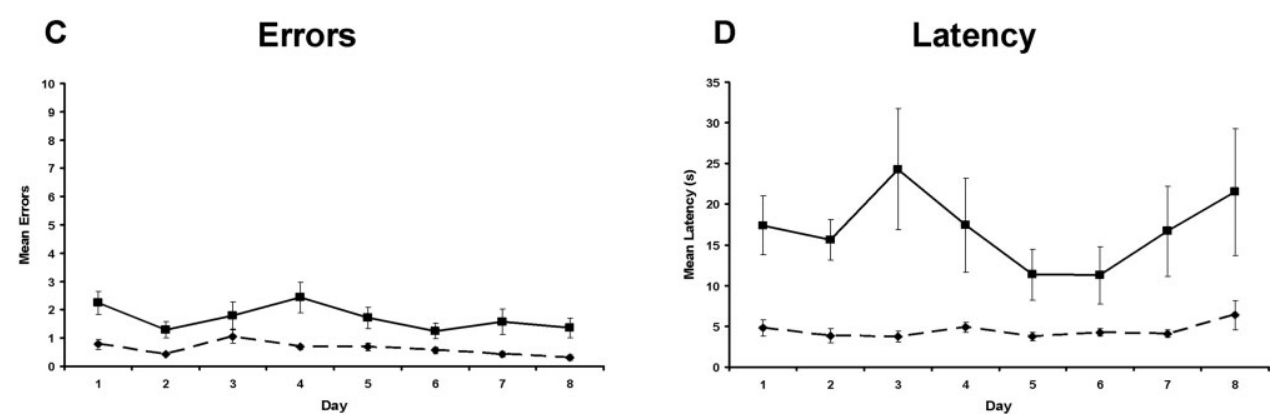

FIGURE 7. Mean numbers of errors and latency to find the food compartment by HPC-R [Pre] and OC-R [Pre] groups in the barrier and no-barrier trials of Experiment 4. The HPC-R [Pre] group performed well in both conditions but, relative to the OC-R [Pre], was impaired on both measures.

ship between the goal box and the local spatial cues in the environment was the same (see Figs. 5A and 7A). The HPC-R [Pre] group consistently performed worse than the OC-R [Pre] group in both conditions (Barrier: Errors- $F_{1,13}=15.87, P<$ 0.002 ; latency- $F_{1,13}=9.18, P<0.01 ;$ No-Barrier: Errors$F_{1,13}=26.73, P<0.0,001$; latency $-F_{1,13}=18.46, P<$ $0.001)$. On the error measure, a significant group $\mathrm{x}$ barrier interaction, $F_{1,13}=6.07, P<0.03$, indicated that the group difference was greater for the barrier than in the no-barrier trials. That interaction was not significant on the latency measure, $F<1$.

Overall, the OC-R [Pre] group encountered barriers slightly more often than the HPC [Pre] group immediately on leaving the start area. This occurred because control rats were more likely to select more direct paths to the food compartment than rats with hippocampal lesions in both the barrier and nobarrier trials. When assessment of the groups' performance was restricted to those barrier trials in which the barrier was directly encountered, the same patterns were observed. The HPC-R [Pre] group made more errors, $F_{1,13}=15.03, P<0.001$, and took longer, $F_{1,13}=18.36, P<0.001$, than the OC-R [Pre] group to find the food compartment.

An important measure of the groups' flexibility in using spatial cues in the familiar environment, was the probability of each rat selecting the most direct route to the food compartment, after encountering a barrier. Rats with hippocampal lesions were relatively inefficient on this measure over the 4 days of barrier trials, selecting the most direct route only $34 \%$ of the time, as compared with a $61 \%$ success rate for the control group, $F_{1,13}=33.84, P<0.0001$.

The results of Experiment 4 show that, notwithstanding the demonstrated ability of preoperatively reared rats with hippocampal lesions to remember specific locations in the village, their spatial memory is susceptible to disruption. Despite the availability of all the original spatial cues, which maintained a constant relationship with the goal box, when confronted with a barrier that blocked their selected route, the HPC-R [Pre] group made more errors and took longer to locate the goal box than normal rats treated in the same way. Although not as severe, this deficit is reminiscent of the HPC-R [Pre] group's impairment on the room-change and village-rotation probe tests (Experiment 3) -in all these cases, preoperatively reared hippocampal rats exhibited inflexibility in their behavior in that they had difficulty adjusting to changes in their environment. In the room-change and village-rotation tests, they were deficient in learning new spatial relationships (re-mapping); in the blocked-route task, the HPC-R [Pre] group was inflexible in its use of learned spatial relationships. This inflexibility may be a symptom of the hippocampally lesioned rat's reliance on schematic spatial representations which do not lend themselves readily to forming new spatial relationships or adjusting to changes in existing representations. 
The results also show that hippocampal lesions affected performance on the no-barrier trials as well as the barrier trials. By comparison, the control animals performed at near-perfect levels on both sets of trials. The HPC-R [Pre] group's impaired performance in the no-barrier trials may be another manifestation of lesion-induced inflexibility. The lesioned group's relatively poor performance may reflect generalized distraction from the barrier trials and an expression of the well-established susceptibility of rats with hippocampal lesions to interfering influences (Douglas, 1967; Kimble, 1968; Winocur and Mills, 1969). Interference from the barrier trials may have challenged the HPC-R [Pre] group's ability to use spatial cues effectively because they relied only on a schematic representation, which may have been perturbed by the barriers. By contrast, control animals were able to adjust to the barrier by virtue of their more detailed, spatial representation which could easily accommodate the distraction introduced by the barrier trials.

A comparison of the number of errors made by the HPC-R [Pre] group on the barrier and no-barrier trials on day 1 provides some support for the interference interpretation. If interference from the barrier trials affected that group's general performance, the effect should be minimal on the first no-barrier trial, which had been preceded by only one barrier trial. In fact, on the first No-barrier trial, the HPC-R [Pre] group made an average of 0.43 errors, as compared with 4.14 errors on the preceding barrier trial, $t_{6}=6.13, P<0.001$. On the next no-barrier trial, where presumably there was more interference, the number of errors by the HPC-R [Pre] group increased to 3.86 errors and, through paired-trials $2-4$, there were no longer differences related to the presence or absence of a barrier, all $t$ s $>0.05$.

Despite the HPC-R [Pre] group's difficulties in the barrier and no-barrier trials, it is important to emphasize that they did use allocentric spatial memory to guide their navigation. For example, even when they failed to choose the most direct route after encountering a barrier, their behavior was not trial and error. Rather, it was directed and purposeful, and they rarely made more than one or two mistakes before finding an acceptable route (see Fig. 7). (Conceivably, frustration resulting from their encounters with the barriers may have been a factor in the performance of the HPC-R [Pre] group in the blockedroutes task, but there were no outward signs of agitation). Thus, while the HPC-R [Pre] group was far from normal in its performance on the tests of Experiment 4, they performed much better than the nonreared hippocampally lesioned rats in Experiment 1 even though the latter did not have to contend with the unpredictable presence of barriers to the reward. This is consistent with the view that the preoperatively reared rats with hippocampal lesions had formed and retained spatial representations of the environment, albeit schematic ones, that could be used to guide their behavior. In the "General Discussion" section we elaborate on this notion.

\section{Anatomical Results}

Figure 8 provides (A) a photomicrograph of coronal sections of a representative hippocampal lesion (left) in relation to a
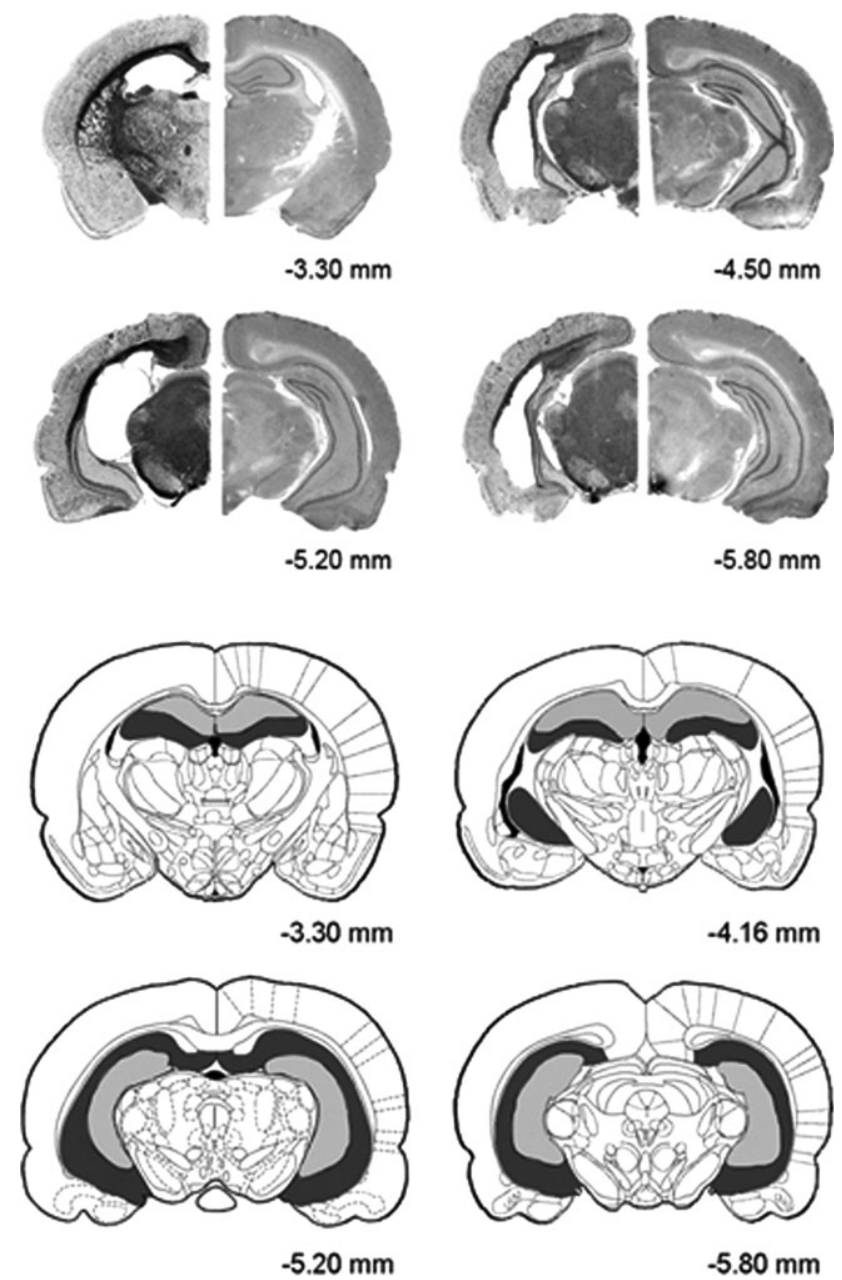

FIGURE 8. (A) Photomicrographs of coronal sections of a representative hippocampal lesion (left) in relation to a normal brain (right) and (B) schematic drawings of minimal (gray) and maximal (black) extents of lesions. The numbers represent the distance in millimeters posterior to bregma.

normal brain (right) and (B) schematic drawings of minimal (gray) and maximal (black) extents of lesions.

The nature and extent of lesions were similar to those reported in recent studies (e.g., Winocur et al., 2005; Winocur et al., 2007). In all the rats with hippocampal lesions that participated in the various experiments, damage extended bilaterally to dorsal and ventral regions of the structure. Twelve of the 33 rats $(36 \%)$ had very large lesions that affected more than $80 \%$ of the hippocampus proper, and in 17 rats $(52 \%), 60$ $80 \%$ of the hippocampus was destroyed. In all of the rats, the lesions included extensive damage to all the subfields (CA1CA3, dentate gyrus). Four rats (16\%) had lesions to $50-60 \%$ of the hippocampus. Overall, the mean value for hippocampal destruction was about $75 \%$, with the extent and pattern of damage to dorsal and ventral regions similar in all groups. In all cases, extra-hippocampal damage was minimal or nonexistent.

ANOVA, performed on the measures of total hippocampal lesion, confirmed that there was no difference in the average volume of hippocampal damage across the three independent 
HPC groups in this study, $F<1$. As well, correlational analyses of the numbers of errors made by rats with hippocampal lesions and the amount of tissue destruction in each rat were performed in the original-environment tests of Experiments 1 and 3, and the blocked-routes test of Experiment 4. These analyses yielded no significant correlations (all $P_{S}>0.05$ ), indicating a lack of relationship between performance and extent of lesion in the present series of experiments.

\section{GENERAL DISCUSSION}

In this study, we showed that there is a marked difference between the effects of pre- and postoperative experience in a complex environment on the formation of spatial memories in rats with hippocampal lesions. Whereas as little as 2 weeks of preoperative rearing was sufficient for rats to develop a representation of the environment that could support accurate navigation and survive hippocampal lesions (Experiment 3), as much as 3 months of postoperative rearing conferred no benefit in comparison with hippocampally lesioned rats that had no rearing experience (Experiment 1). Nonetheless, following specific goal-directed training, rats with hippocampal lesions, with or without postoperative rearing experience in the village, eventually were able to learn the location of food reward and form accurate spatial memories. Probe tests showed that the representations of these memories were similar to those that survived hippocampal lesions in preoperatively reared rats. Moreover, the responses of the pre- and postoperatively lesioned rats to spatial manipulations in the probe tests were similar to those of control rats, suggesting that the lesioned rats' navigation through the village was also guided by allocentric, spatial representations (Experiments 2 and 3). However, a critical difference between the lesioned and control rats was in the flexibility of their spatial memories, as seen in two ways: (1) rats with hippocampal lesions took longer than controls to re-map old representations to new conditions (e.g., village-rotation and roomchange probe tests), usually failing to reach the controls' level of performance; (2) even preoperatively reared rats could not use learned representations effectively when confronted with impediments to their normal navigation (blocked-routes task, Experiment 4).

This pattern of results suggests the following conclusions. First, an intact hippocampus helps in the formation of spatial representations which then can survive hippocampal damage. Second, these extra-hippocampal representations are similar to those formed in rats with hippocampal damage receiving goaldirected training, and are allocentric in nature. Third, the extra-hippocampal representations, though allocentric, are fundamentally different from those that include the hippocampus. The former are more schematic and less cohesive (integrated) than the latter and, consequently, less capable of supporting flexible adjustments to changes in the environment. An important implication of these conclusions is that although the hippocampus contributes to the formation of extra-hippocampal representations in the intact brain, these representations are not duplicates of those that are hippocampally dependent.

The essential question that arises from these conclusions concerns the nature of the hippocampal-dependent and extra-hippocampal spatial representations. Our findings add to the already considerable evidence that the hippocampus is necessary for relational learning that is crucial for the formation of detailed, spatial representations of the environment whose elements are integrated into a cognitive map (O'Keefe and Nadel, 1978; Eichenbaum and Cohen, 2001; Burgess et al., 2002). Such a cognitive map affords rapid place-learning, as well as the flexibility to make adjustments to changes in the environment that require either learning new relationships or using old representations in new ways. What has emerged from our village research is that allocentric, spatial representations can exist independently of the hippocampus and be used for navigation. As Nadel and coworkers $(1980,1985)$ proposed some time ago, these extra-hippocampal representations appear to be comprised of discrete elements that consist of stimuli that potentially could serve as landmarks, or form small ensembles or constellations within the distal environment that provide spatial references for guiding navigation. What distinguishes the representations in the hippocampally damaged brain from those associated with cohesive cognitive maps in the intact brain, is that the former give rise to more schematic memories that consist of elements that are not fully integrated with one another. In other words, in the schematic spatial memories that are available to rats with hippocampal damage, the relationships or links among the component elements are not specified as part of a coherent representation. By contrast, representations of spatial memory that involve the hippocampus consist of a rich network of linkages among the elements that indicate clearly how each is related to the other, thereby permitting efficient learning and flexible responses to spatial challenges. In this study, we showed that (1) when spatial memories are formed in the presence of the hippocampus, only the schematic versions, made up of unlinked ensembles of elements, survive damage to the structure, and (2) through extensive training, rats with hippocampal lesions can laboriously form schematic spatial memories that exhibit the same characteristics as those formed preoperatively (see Fig. 9 for an imaginary visual rendering of spatial representations in intact and hippocampally damaged rat brains).

This framework accounts both for the pattern of preserved and impaired memory seen in rats with hippocampal lesions, regardless of whether memory acquisition occurred preoperatively or postoperatively. Once the schematic representation is acquired, allocentric spatial cues in the original environment can support navigation, even when the number of available, salient cues has been significantly reduced. Working with an ensemble of stimuli that represents only a limited part of the environment that is not linked to other parts of it the hippocampally damaged rat does not know the relation of that ensemble to others and, therefore, cannot easily find the most direct alternative route when, for example, confronted by a barrier. At the same time, performance is not necessarily random because the rat is capable of reorienting itself to new spatial 


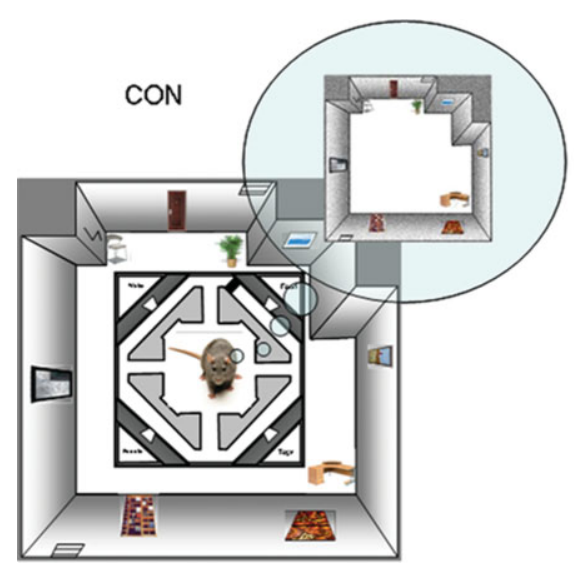

FIGURE 9. A cartoon schema of how the village, as a familiar visual environment, is represented in the brains of intact rats and rats with hippocampal lesions. In the intact brain, all the spatially distributed elements in the environment and their relationships to each other are represented holistically as part of a cohesive picture. The elements are highly integrated and, by virtue of the connections between them, normal rats can readily access different combinations to serve as reference points and guide behavior, depending on task demands (Tolman, 1948; O'Keefe and Nadel, 1978). In the hippocampally damaged brain, the familiar environment is represented as a collection of separate and weakly connected

coordinates and uses that reference point to find another, usually less direct, route to the goal (see Fig. 6). Similarly, in the room-change and village-rotation probe tests, where efficient performance depended on an integrated representation of the spatial environment and new relational learning, rats with hippocampal lesions were predictably handicapped, although they were able to benefit from the availability of schematic spatial memories.

As indicated earlier, the schematic memories that were acquired preoperatively and survived hippocampal lesions share characteristics with those formed postoperatively after extensive training. However, it is clear that different processes are involved in their formation. For example, mere exposure prior to hippocampal damage is sufficient to develop the schematic representation, suggesting that it benefits from the availability of a hippocampally dependent cognitive map. By comparison, in the hippocampally damaged rat, extensive goal-directed training is required to achieve the same representation. This suggests the possibility that, in the intact brain, the schematic representation derives from the integrated representation of elements that comprises the cognitive map. This could be accomplished through a transformation process that allows for the abstraction of those local elements that are needed for navigation, but without the specific links among them.

We view the hippocampal and extra-hippocampal representations used by rats for navigation as comparable with representations used by humans for navigation and for constructing and reconstructing complex memories. In an interesting approach, Hassabis et al. (2007) noted that, relative to healthy controls, medial temporal-lobe amnesics are impaired in describing imaginary scenes. The amnesics' scenes lack detail and spatial coherence, precisely those properties that were deficient in our hippocampal rats. Amnesics described their imagined scenes as a series of separate components and isolated snapshots, rather than coherent, integrated wholes. In that sense, the scene construction deficits described by Hassabis et al. (2007) resemble the spatial deficits of our hippocampally damaged rats, with both relying on schematic memories made up of fragmented components lacking integration.

Humans with a deficit in scene construction, like our hippocampally damaged rats, would be expected to have preserved spatial memories acquired in the remote past as long as they could rely on schematic representation of local elements and did not require detailed, integrated representations for successful performance. Here, the evidence is equivocal. While it is known that the spatial memories of amnesic patients suffer from a paucity of perceptual detail (Rosenbaum et al., 2000), it is less clear whether their schematic representations of the environment are sufficient for navigation under most circumstances. There is certainly evidence that amnesic patients can navigate normally in a highly familiar environment (Beatty et al., 1987; Teng and Squire, 1999; Rosenbaum et al., 2000; Corkin, 2002) and even deal with detours if the most direct route is blocked (Rosenbaum et al., 2000). However, such abilities, presumably dependent on a schematic representation, may be preserved only when the spatial layout is relatively simple and grid-like, as in the street layout of a city like Toronto. When the connections between one location and another are more complex, as in the tortuous streets of London, performance may break down, suggesting that for purposes of spatial navigation, schematic representations have distinct limitations (Spiers and Maguire, 2006). Even with respect to simple environments, in at least one amnesic patient, 
we observed a lack of appreciation of the relation of one location to another. When facing in one direction and looking at a particular landmark in a highly familiar environment, the patient had difficulty describing what was behind him, though healthy controls found this relatively easy (Rosenbaum et al., unpublished observation).

The present data are consistent with the results of our previous village studies in suggesting that rats with hippocampal lesions, following preoperative rearing or extensive postoperative training, adopt a spatial strategy based on the use of allocentric spatial cues. An alternative explanation is that the lesioned rats were following a nonspatial, response-based strategy that involved learning relationships between discrete stimuli and goal-directed responses. Work by Packard et al. (1989) and Packard and McGaugh (1992) showed that, while hippocampus-dependent spatial or place-learning is a default strategy for normal rats, they also engage in stimulus-response learning under the control of a striatal system that includes the caudate nucleus. Indeed, if either system is abolished after extensive training in which both types of learning have occurred, rats are likely to revert to the strategy associated with the surviving system (Packard and McGaugh, 1996; see also Iaria et al., 2003). Thus, it is conceivable that rats with hippocampal lesions used the striatal system and a form of stimulus-response learning to locate the food in our village tests.

Although the alternative view can explain the savings exhibited by preoperatively reared hippocampal groups, and the ability of rats with hippocampal lesions without preoperative experience to learn eventually to find the food reward, it cannot account for critical findings in the probe tests. The use of a stimulus-response strategy depends on the identification of discrete stimuli, internal or external to the village, which are aligned with the goal box and can be associated with responses that will lead to the goal box. In the floor-rotation test, where relationships between internal stimuli and the goal box were disrupted, rats with hippocampal lesions would be expected to be severely impaired if they depended on such relationships. While differences between HPC and control groups, in terms of numbers of errors, were sometimes seen on this test (Experiments 2 and 3), in real terms the differences were negligible and more likely due to nonspecific factors. In other experiments (Winocur et al., 2005; Winocur et al., in press), the typical finding was no difference on the floor-rotation test. In the cue-distortion test, most of the environmental cues, and all within close range of the village, were relocated or removed, so any learning based on fixed, external landmarks or stimulus-response relationships should be disrupted. However, preoperatively reared rats with hippocampal lesions that had demonstrated excellent savings when tested in the original environment, continued to perform as well when the cues were re-arranged. The results of the cue-distortion test show that schematic memories do not depend on integrated relationships between specific cues. Rather, they seem to depend on a minimal configuration of cues (in this case, perhaps local features of the room's geometry) that bears a constant relationship to specific locations and is capable of supporting limited navigation within the environment.
Previously, we referred to the extra-hippocampal representation of spatial memory as simply a rudimentary, coarser version of the cohesive, integrated representation that forms with the hippocampus intact (Winocur et al., 2005). Such a representation could account for the excellent performance of rats with hippocampal lesions in the original-environment test or even in the cue-distortion probe test when only a few, familiar cues were available (Experiments 2 and 3; Winocur et al., 2005; Winocur et al., in press). If the representation merely lacked perceptual detail, a decline in performance on the blocked-routes test would not be expected in hippocampal rats since the details available to guide behavior were at least as plentiful as in the cue-distortion test. Their impaired performance on the blocked-routes test indicates that not only does the representation lack perceptual detail, but it also lacks linkages among the elements.

The present results do not challenge the well-established view that the hippocampus is critical to the process of efficient spatial learning and memory (O'Keefe and Nadel, 1978). They do, however, underscore the point that spatial memories, formed with the involvement of the hippocampus, can survive damage to the structure, but in a different form. In short, it is not the case that, with the passage of time, hippocampal representations are duplicated in extra-hippocampal structures as standard views of consolidation would assert. Hippocampal and extra-hippocampal spatial memories are fundamentally different, no matter when they were formed. Although the latter memories can support accurate place finding under certain conditions, they are generally less efficient, and appear to have different characteristics.

Finally, interesting comparisons can be drawn between the present results and findings related to the effects of hippocampal lesions on contextual fear conditioning. Rodents with hippocampal lesions are impaired in recalling a preoperatively acquired contextual fear response if the lesion is made shortly after conditioning (Kim and Fanselow, 1992) but the lesions do not necessarily affect the learning of a contextual fear response (Phillips and LeDoux, 1994; Frankland et al., 1998; Gisquet-Verrier et al., 1999; Moses et al., 2007). In the latter case, however, it does not appear that the contextual fear response learned by animals with hippocampal damage is the same as that learned by normal animals. For example, various reports indicate that the fear response learned by animals with hippocampal damage (1) is weaker, as evidenced by less time spent freezing (Phillips and LeDoux, 1994), (2) lacks contextspecificity (Frankland et al., 1998), (3) requires additional conditioning trials to become established (Wiltgen et al., 2006), and (4) depends on the training procedures (Moses et al., 2007). These findings are generally consistent with the view that hippocampally damaged animals are impaired in forming the necessary relationships to support rapid contextual fear conditioning. In the absence of the hippocampus, such learning is accomplished by less efficient neocortical mechanisms that give rise to imprecise memories that may be characterized as general or schematic in nature. In a recent review, Rudy (2009) attributed poor relational learning following hippocampal damage to a lack of the integrative properties necessary to perform pattern completion (see also Nakazawa et al., 2002). This notion reso- 
nates with the idea, expressed in this article, that the representation of spatial memory in rats with hippocampal lesions lacks the integrated neural linkages that are necessary for efficient navigation in a complex environment. Clearly, there is work to be done in specifying the role of the hippocampus in forming context-dependent memories, and in characterizing the nature of schematic memories that are represented in extra-hippocampal structures, but the parallels that are emerging in the animal and human literatures reinforce the generality of the present findings.

\section{Acknowledgments}

The authors gratefully acknowledge Dr. Malcolm Binns for his help in analyzing the data, as well as Andrea Goode, Jason Allen, and Lucy McGarry for their technical assistance. They also thank an anonymous reviewer for drawing the attention to the parallels between the results obtained from this study and those related to contextual fear conditioning.

\section{REFERENCES}

Beatty WW, Salmon DP, Bernstein N, Butters N. 1987. Remote memory in a patient with amnesia due to hypoxia. Psychol Med 17:657-665.

Burgess N, Maguire EA, O'Keefe J. 2002. The human hippocampus and spatial and episodic memory. Neuron 35:625-641.

Corkin S. 2002. What's new with the amnesic patient H.M.? Nat Rev Neurosci 3:153-160.

Douglas RJ. 1967. The hippocampus and behavior. Psychol Bull 67:416-442.

Eichenbaum H, Cohen N. 2001. From Conditioning to Conscious Recollection: Memory Systems of the Brain. New York: Oxford University Press.

Frankland PW, Cestari V, Filipkowski RK, McDonald RJ, Silva AJ. 1998. The dorsal hippocampus is essential for context discrimination but not for contextual conditioning. Behav Neurosci 112:863-874.

Gisquet-Verrier P, Dutrieux G, Richer P, Doyere V. 1999. Effects of lesions to the hippocampus on contextual fear: Evidence for a disruption of freezing and avoidance behavior but not context conditioning. Behav Neurosci 113:507-522.

Hassabis D, Kumaran D, Vann SD, Maguire EA. 2007. Patients with hippocampal amnesia cannot imagine new experiences. Proc Natl Acad Sci USA 104:1726-1731.

Hebb DO. 1938. Studies of the organization of behaviour. I. Behaviour of the rat in field orientation. J Comp Psychol 25:333-352.

Iaria G, Petrides M, Dagher A, Pike B, Bohbot VD. 2003. Cognitive strategies dependent on the hippocampus and caudate nucleus in human navigation: Variability and change with practice. J Neurosci 23:5945-5952.

Jarrard LE, Meldrum BS. 1993. Selective excitotoxic pathology in the rat hippocampus. Neuropath Appl Neurobiol 19:381-389.

Kim JJ, Fanselow MS. 1992. Modality-specific retrograde amnesia of fear. Science 256:675-677.

Kimble DP. 1968. Hippocampus and internal inhibition. Psychol Bull 70:285-295.

Maguire EA, Nannery R, Spiers HJ. 2006. Navigation around London by a taxi driver with bilateral hippocampal lesions. Brain 129 (Part 11):2894-2907.
Morris RG, Garrud P, Rawlins JN, O'Keefe J. 1982. Place navigation impaired in rats with hippocampal lesions. Nature 297:681-683.

Moses SN, Winocur G, Ryan JD, Moscovitch M. 2007. Environmental complexity affects contextual fear conditioning following hippocampal lesions in rats. Hippocampus 17:333-337.

Nadel L, Willner J. 1980. Context and conditioning: A place for space. Physiol Psychol 8:218-228.

Nadel L, Willner J, Kurz EM. 1985. Cognitive Maps and Environmental Context. Context and Learning. New Jersey: Lawrence and Erlbaum and Associates. pp 385-406.

Nakazawa K, Quirk MC, Chitwood RA, Watanabe M, Yeckel MF, Sun LD, Kato A, Carr CA, Johnston D, Wilson MA, Tonegawa S. 2002. Requirement for hippocampal CA3 NMDA receptors in associative memory recall. Science 297:211-218.

O'Keefe J, Nadel L. 1978. The Hippocampus as a Cognitive Map. Oxford: Oxford University Press.

Olton DS, Becker JT, Handelmann GE. 1979. Hippocampus, space, and memory. Behav Brain Sci 2:313-365.

Packard MG, McGaugh JL. 1992. Double dissociation of fornix and caudate nucleus lesions on acquisition of two water maze tasks: Further evidence for multiple memory systems. Behav Neurosci 106:439-446.

Packard MG, McGaugh JL. 1996. Inactivation of hippocampus or caudate nucleus with lidocaine differentially affects expression of place and response learning. Neurobiol Learn Mem 65:6572.

Packard MG, Hirsh R, White NM. 1989. Differential effects of fornix and caudate nucleus lesions on two radial maze tasks: Evidence for multiple memory systems. J Neurosci 9:1465-1472.

Paxinos G, Watson C. 1997. The Rat Brain in Stereotaxic Coordinates. New York: Academic Press.

Phillips RG, LeDoux JE. 1994. Lesions of the dorsal hippocampal formation interfere with background but not foreground contextual fear conditioning. Learn Mem 1:34-44.

Rosenbaum RS, Priselac S, Kohler S, Black SE, Gao F, Nadel L, Moscovitch M. 2000. Remote spatial memory in an amnesic person with extensive bilateral hippocampal lesions. Nat Neurosci 3:10441048.

Rudy JW. 2009. Context representations, context functions, and the parahippocampal-hippocampal system. Learn Mem 16:573-585.

Spiers HJ, Maguire EA. 2006. Thoughts, behaviour, and brain dynamics during navigation in the real world. Neuroimage 31:18261840.

Teng E, Squire LR. 1999. Memory for places learned long ago is intact after hippocampal damage. Nature 400:675-677.

Tolman EC. 1948. Cognitive maps in rats and man. Psychol Rev 55:189-208.

Wiltgen BJ, Sanders MJ, Anagnostaras SG, Sage JR, Fanselow MS 2006. Context fear learning in the absence of the hippocampus. J Neurosci 26:5484-5491.

Winocur G. 1982. Radial-arm-maze behavior by rats with dorsal hippocampal lesions: Effects of cueing. J Comp Physiol Psychol 96:155-169.

Winocur G, Mills JA. 1969. Hippocampus and septum in response inhibition. J Comp Physiol Psychol 67:352-357.

Winocur G, Moscovitch M, Fogel S, Rosenbaum RS, Sekeres M. 2005. Preserved spatial memory after hippocampal lesions: Effects of extensive experience in a complex environment. Nat Neurosci $8: 273-275$.

Winocur G, Moscovitch M, Sekeres M. 2007. Memory consolidation or transformation: Context manipulation and hippocampal representations of memory. Nat Neurosci 10:555-557.

Winocur G, Moscovitch M, Rosenbaum RS, Sekeres M. A study of remote spatial memory in aged rats. Neurobiol Aging (in press). 